\section{Primary amyloidosis presenting as an isolated mediastinal mass: diagnosis by fine needle biopsy}

\author{
N Hiller, D Fisher, O Shmesh, \\ S Gottschalk-Sabag, M Dollberg
}

\begin{abstract}
Intrathoracic amyloidosis affecting the lungs or mediastinum is rare, and mediastinal lymphadenopathy in the absence of pulmonary involvement is even more rare. The case history is presented of a previously healthy man who developed nodular mediastinal amyloidosis without pulmonary involvement. Diagnosis was made by percutaneous fine needle biopsy. (Thorax 1995:50:908-909)
\end{abstract}

Keywords: amyloidosis, percutaneous fine needle biopsy.

Primary systemic amyloidosis is an uncommon dysproteinaemia, and intrathoracic involvement is one of the rarest forms. Mediastinal lymphadenopathy without peripheral lymph node enlargement and lung parenchymal involvement is even more rare. ${ }^{1-4}$

We present a case in which a mediastinal mass was the sole manifestation of primary amyloidosis. The diagnosis was made by a CTguided fine needle biopsy.

\section{Case report}

A 66 year old Ashkenazi Jewish man was admitted to hospital after two weeks of dyspnoea,

cough, and fatigue. There were no associated fever, chills, or night sweats. His medical history included non-insulin dependent diabetes mellitus with secondary retinal damage. Physical examination was unremarkable apart from some ankle oedema. All laboratory tests were within normal limits.

Routine chest radiography on admission revealed a right hilar mass and a mass in the posterior mediastinum, with a right pleural effusion (fig 1). A computed tomographic (CT) scan of the chest demonstrated an homogeneous lobulated mass occupying the posterior mediastinum, bilateral hilar lymphadenopathy more pronounced on the right side, and bilateral pleural effusions (fig 2). Further investigations including abdominal ultrasonography, gallium scan, and bone marrow biopsy were normal. Thoracocentesis yielded $400 \mathrm{ml}$ of bloody fluid with was rich in lymphocytes but contained no neoplastic cells. Bronchoscopy showed extrabronchial obstruction of the right middle lobe bronchus but no endobronchial lesion. Bronchial brushing and biopsy samples were not diagnostic. Percutaneous needle biopsy of the mediastinal mass was performed and microscopic examination showed lymphocytes, giant cells, epithelioid cells, and amorphous eosinophilic substance which stained positive with Congo red. Under polarised light microscopy Congo red-stained material showed the characteristic green birefringence of amyloidosis. Rectal biopsy performed later was also positive for amyloid disease.

\title{
Discussion
}

Amyloidosis is an uncommon diverse pathological process resulting from the deposition of an inert eosinophilic proteinaceous material in tissues. The disease might be primary or hereditary, but most cases are secondary. Intrathoracic involvement is more common in primary amyloid disease. ${ }^{5}$

Up to $37 \%$ of cases of generalised primary and secondary amyloidosis have lymph node involvement. ${ }^{2}$ It has been suggested that an unidentified localising factor is responsible for organ predilection in systemic amyloidosis which spares the intrathoracic lymph nodes. ${ }^{6}$ The radiographic features of intrathoracic amyloidosis have been based upon the description of a limited number of cases. Naschitz et $a^{2}$ reviewed 13 cases of intrathoracic lymphadenopathy due to amyloidosis and found that the lung parenchyma was not involved in only three cases. Peripheral lymph node enlargement was recorded in all these patients.

According to Wilson $\mathrm{et} \mathrm{al}^{4}$ coarse calcification seen on CT scanning is a frequent feature of mediastinal lymphadenopathy in amyloidosis. Jenkins and Potter ${ }^{1}$ reviewed the literature and found only four reported cases of pseudotumoral mediastinal amyloidosis. Calcification was present in $50 \%$ of cases.

The diagnosis of amyloidosis was achieved in all the cases reviewed by mediastinoscopy, open biopsy, or postmortem examination. ${ }^{2}$ Percutaneous fine needle biopsy can be performed

Figure 1 Posteroanterior chest radiograph showing enlargement of right hilum and right pleural effusion. 


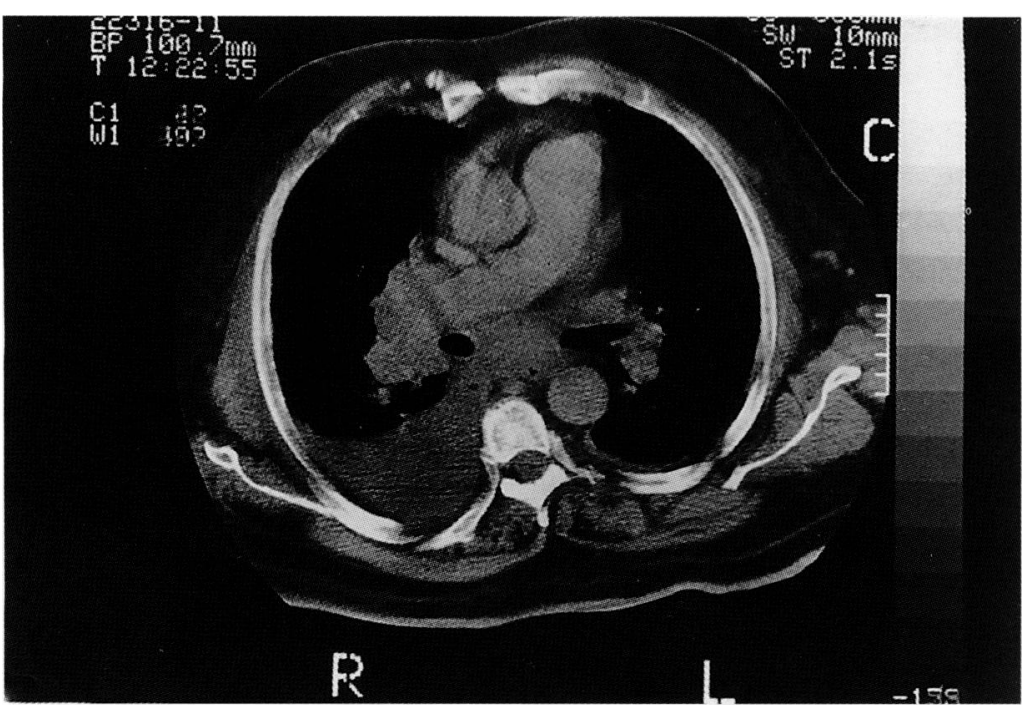

Figure 2 Contrast enhanced computed tomographic scan of the chest showing bilateral hilar adenopathy, posterior mediastinal adenopathy, and bilateral pleural effusion. only when the patient is cooperative and has no bleeding diathesis. It is usually an easy, efficient and safe procedure.

Mediastinal amyloidosis occurs infrequently and may be confused with other tumours more usually found in this region. The diagnosis should be considered in the evaluation of a large mediastinal mass. Percutaneous needle biopsy may be sufficient to provide a definitive diagnosis.

1 Jenkins NCF, Potter M. Calcified pseudotumoral mediastinal amyloidosis. Thorax 1991;46:686-7.

2 Naschitz JE, Yeshurun D, Pick AI. Intrathoracic amyloid lymphadenopathy. Respiration 1986;49:73-6.

3 Osnoss KL, Harell DD. Isolated mediastinal mass in primary amyloidosis. Chest 1980;78:786-8.

4 Wilson SR, Sanders DE, Delarue NC. Intrathoracic manifestations of amyloid disease. Radiology 1976;120:283-9. 5 Celli BR, Rubinow A, Cohen AS, et al. Patterns of pulmonary involvement in systemic amyloidosis. Chest 1978;74:543-7. 6 Franklin EC. Some unresolved problems in amyloid disease. Am F Med 1979;66:365-7.

\section{Adult congenital lobar emphysema in pregnancy}

\author{
Paul S Critchley, \\ Christopher P Forrester-Wood, \\ Paul D Ridley
}

\begin{abstract}
A young woman presented with left sided chest pain. Chest radiography revealed a hyperexpanded left upper lobe and the rare diagnosis of congenital lobar emphysema was made. She was then found to be pregnant. Thoracotomy and left upper lobectomy were performed during the pregnancy without adverse effects to the mother or fetus. The implications of pregnancy on the surgical management of this rare condition are discussed.

(Thorax 1995;50:909-910)
\end{abstract}

Department of

Thoracic Surgery,

Frenchay Hospital, Bristol BS16 1LE, UK

P S Critchley

C P Forrester-Wood P D Ridley

Reprint requests to: Dr P S Critchley.

Received 31 March 1994 Returned to authors 8 June 1994

Revised version received 26 August 1994 Accepted for publication 5 September 1994
Keywords: congenital lobar emphysema, pregnancy.

An 18 year old woman presented to her family doctor with left sided chest pain. This followed a minor coughing episode, although inhalation well. The chest radiograph revealed hyperexpansion of the left upper lobe with mediastinal shift (fig 1). Computed tomographic scanning showed an emphysematous left upper lobe (fig 2). Respiratory function tests revealed was denied. She had previously been fit and a forced expiratory volume in one second $\left(\mathrm{FEV}_{1}\right)$ of 1.71 and a forced vital capacity (FVC) of $2 \cdot 11$, both $50 \%$ of her predicted values. She was therefore referred to our thoracic unit for bronchoscopy in view of the possibility of an obstructing foreign body. At this stage she was found to be six weeks into her first pregnancy. Subsequent bronchoscopy failed to show an obstructing endobronchial lesion and diagnosis of congenital lobar emphysema was made. As the pregnancy progressed she became increasingly dyspnoeic and the chest radiograph showed further mediastinal shift.

At 26 weeks gestation a thoracotomy and left upper lobectomy were performed. The operative procedure consisted of a standard

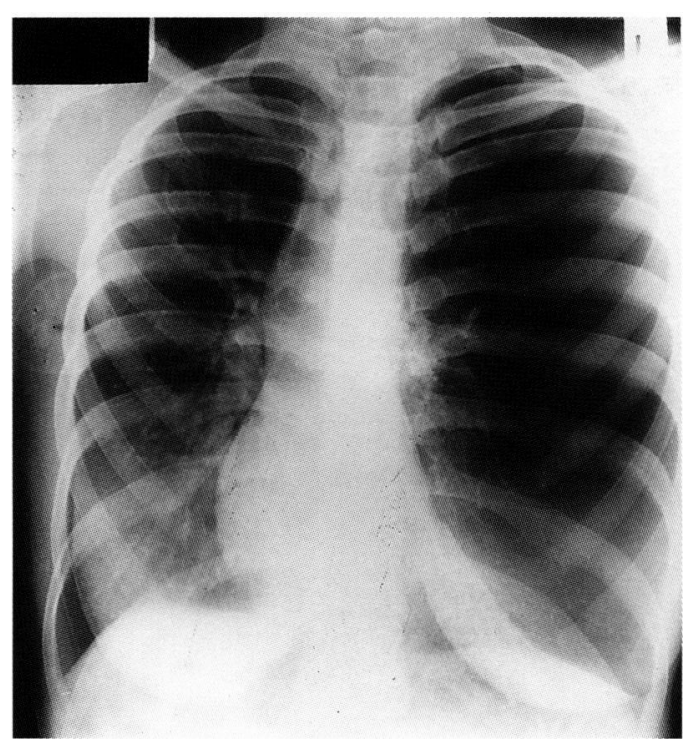

Figure 1 Chest radiograph at presentation showing hyperlucent left upper lobe with mediastinal shift toward the right. 